\title{
RECO Flow Restoration Device Versus Solitaire FR With the Intention for Thrombectomy Study (REDIRECT): a prospective randomized controlled trial
}

\author{
Jie Cao, MD, ${ }^{1}$ Hang Lin, MS, ${ }^{2}$ Min Lin, MS, ${ }^{2}$ Kaifu Ke, MD, ${ }^{3}$ Yunfeng Zhang, MD, ${ }^{3}$ Yong Zhang, MS, ${ }^{4}$ \\ Weihong Zheng, MA, ${ }^{5}$ Xingyu Chen, MA, ${ }^{5}$ Wei Wang, MD, ${ }^{6}$ Meng Zhang, MD, ${ }^{7}$ Jinggang Xuan, MA, ${ }^{1}$ \\ and Ya Peng, MD, ${ }^{1}$ for the REDIRECT Trial Investigators
}

\begin{abstract}
1'Department of Neurosurgery, The First People's Hospital of Changzhou/The Third Affiliated Hospital of Soochow University, Changzhou; ' ${ }^{D}$ epartment of Neurology, Fuzhou General Hospital of Nanjing Military Command, Fuzhou; ${ }^{3}$ Department of Neurology, The Affiliated Hospital of Nantong University, Nantong; ‘ Department of Neurology, The Affiliated Hospital of Qingdao University, Qingdao; ${ }^{5}$ Department of Neurology, Zhongshan Hospital of Xiamen University, Xiamen; ${ }^{6}$ Department of Imaging, Yangzhou No. 1 People's Hospital, Yangzhou; and 'Department of CCU, Daping Hospital, Chongqing, People's Republic of China
\end{abstract}

OBJECTIVE The RECO flow restoration (FR) device is a new stent retriever designed for rapid flow restoration in acute ischemic stroke (AIS) caused by large vessel occlusion (LVO). Here, the authors compared the efficacy and safety of the RECO device with the predicate Solitaire FR stent retriever.

METHODS The RECO Flow Restoration Device Versus Solitaire FR With the Intention for Thrombectomy Study (REDIRECT) was a multicenter, prospective, open randomized controlled trial. Patients with acute LVO at 7 Chinese stroke centers participated in the study. The primary efficacy endpoint was defined as a modified thrombolysis in cerebral infarction $(\mathrm{mTICl})$ reperfusion grade $\geq 2$ within three passes. The primary safety endpoint comprised any serious adverse device effect, symptomatic intracerebral hemorrhage $(\mathrm{sICH})$, and any serious adverse event (SAE; defined as cerebral palsy or death) within 24 hours after the procedure. The secondary efficacy endpoints consisted of functional independence (modified Rankin Scale score 0-2), procedure duration, and 90-day all-cause mortality.

RESULTS Between January 2014 and August 2016, 67 patients were randomly allocated to the RECO group and 69 patients to the Solitaire FR group. The primary efficacy endpoint ( $\mathrm{mTICl}$ grade $\geq 2$ within three passes) was similar in the two treatment groups ( $91 \%$ vs $87 \%$, respectively, $p=0.5861$ ), and the rate of reperfusion with an $\mathrm{mTICl}$ grade $2 \mathrm{~b} / 3$ was $87 \%$ versus $75 \%(p=0.1272)$. There were no serious adverse device effects in any patient. The rates of sICH $(1.5 \%$ vs $7.2 \%, p=0.1027)$ and SAEs $(6.0 \%$ vs $1.4 \%, p=0.2050)$ within 24 hours after the procedure were similar in the two treatment groups. There was no significant difference in the rate of functional independence $(63 \% \mathrm{vs} 46 \%, p=0.0609)$ or 90 -day all-cause mortality ( $13 \%$ vs $23 \%, p=0.1848)$ or in procedure duration ( $85.39 \pm 47.01$ vs $89.94 \pm 53.34$ minutes, $p=0.5986)$ between the two groups.

CONCLUSIONS The RECO stent retriever is effective and safe as a mechanical thrombectomy device for AIS due to LVO. Clinical trial registration no.: NCT01983644 (clinicaltrials.gov)

https://thejns.org/doi/abs/10.3171/2020.3.JNS193356

KEYWORDS intracranial large vessel occlusion; mechanical thrombectomy device; revascularization; symptomatic intracerebral hemorrhage; vascular disorders

$\mathrm{M}$ ECHANICAL thrombectomy (MT) has been proven successful for revascularization through recent randomized controlled trials (RCTs) on the treatment of acute ischemic stroke (AIS) due to large vessel occlusion (LVO) $\cdot{ }^{1-5}$ It is related to good clinical outcomes and decreased disability and mortality rates. ${ }^{6}$ Among the various MT techniques, stent retriever thrombectomy has recently become widely used. MT with stent retrievers for intracranial LVO has been associated with faster and higher rates of reperfusion. ${ }^{7}$ In recent clinical studies, stent

ABBREVIATIONS AIS = acute ischemic stroke; FR = flow restoration; LVO = large vessel occlusion; $\mathrm{mRS}=$ modified Rankin Scale; $M T$ = mechanical thrombectomy; $\mathrm{mTICl}=$ modified thrombolysis in cerebral infarction; NIHSS = National Institutes of Health Stroke Scale; RCT = randomized controlled trial; rt-PA = recombinant tissue plasminogen activator; $\mathrm{SAE}=$ serious adverse event; $\mathrm{sICH}=$ symptomatic intracerebral hemorrhage.

SUBMITTED December 12, 2019. ACCEPTED March 17, 2020.

INCLUDE WHEN CITING Published online June 5, 2020; DOI: 10.3171/2020.3.JNS193356. 


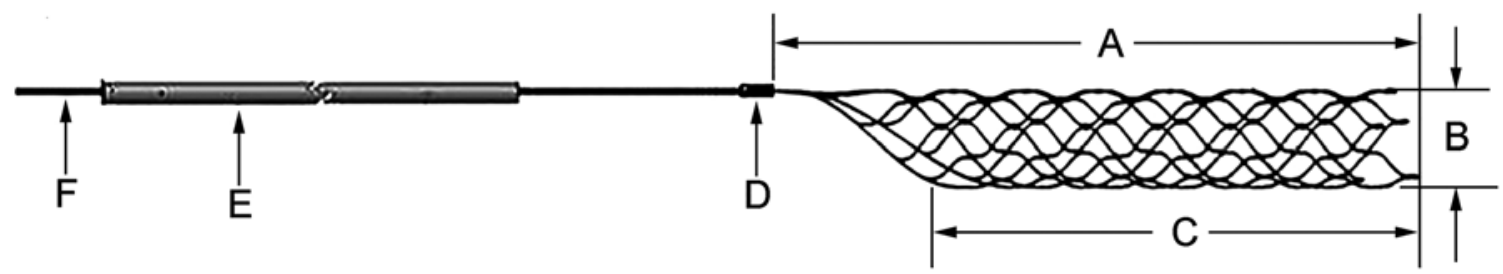

FIG. 1. Description of stent composition: total length of stent (A), diameter of stent (B), length of stent available for use (C), radiopaque markers $(D)$, protection sheath $(E)$, and pushing wire $(F)$.

retriever thrombectomy has shown good recanalization rates, varying from $67 \%$ to $94 \% .8,9$

The Solitaire FR With the Intention for Thrombectomy (SWIFT) study ${ }^{10}$ and Thrombectomy Revascularization of Large Vessel Occlusions in Acute Ischemic Stroke (TREVO 2) trial $^{11}$ first declared that MT with the Solitaire flow restoration (FR) stent (ev3 Covidien) and the Trevo stent (Stryker Neurovascular) achieved better revascularization than that with the Merci retriever device. It has been shown that both the Trevo and Solitaire stent retrievers have a higher revascularization rate and better clinical prognosis than those obtained with the Merci device, while the safety profiles are similar. ${ }^{3}$ In addition, a metaanalysis of 19 studies revealed that the Trevo and Solitaire stents have similar efficacy and safety, although the mortality rate with the Solitaire stent is lower..$^{12}$ Furthermore, a meta-analysis of previous RCTs comparing MT devices (Trevo, Solitaire, Aspiration) found that the Trevo and Solitaire stents have a greater likelihood of better prognosis, while the Solitaire stent and Aspiration devices appear to be safer. ${ }^{13}$

The RECO FR device (Minitech Medical) is a selfexpanding clot retriever designed for FR in patients with ischemic stroke caused by intracranial LVO. The device is used for neurovasculature of the intracranial large artery. It is a stent retriever including a self-expanding clotretrieving stent, a pushing wire connected to the stent, and a protection sheath (Fig. 1).

The RECO Flow Restoration Device Versus Solitaire FR With the Intention for Thrombectomy Study (REDIRECT) was a multicenter, prospective, open RCT. The aim of the trial was to confirm the efficacy and safety of the RECO stent retriever for MT in patients with acute intracranial LVO compared with the Solitaire FR stent.

\section{Methods}

\section{Study Design and Participants}

REDIRECT was a multicenter, prospective, open RCT, and it was registered with clinicaltrials.gov (clinical trial registration no.: NCT01983644). The study was authorized by the ethics committee of each participating medical center as well as the appropriate national regulatory bodies. Every enrolled patient or his or her legally authorized representative was informed and agreed to participation. Consents were obtained prior to performing the thrombectomies. The key inclusion criteria were as follows: 1) patient age 18-80 years; 2) AIS within 8 hours after symptom onset with a baseline National Institutes of Health Stroke Scale (NIHSS) score $\geq 8$ and $\leq 24$; 3 ) angi- ographically proven occlusion (modified thrombolysis in cerebral infarction [mTICI] reperfusion grade $\leq 1$ ) of the intracranial large artery and the ability to receive endovascular treatment (no less than one RECO or Solitaire stent deployment) within 8 hours after symptom onset; and 4) treatment with intravenous recombinant tissue plasminogen activator (rt-PA; if appropriate, within 4.5 hours after symptom onset).

The key exclusion criteria were as follows: 1) intracranial hemorrhage or tumor proved by CT or MRI; 2) serious ischemic infarction (acute ischemic change in over a third of the middle cerebral artery territory); 3) serious sustained hypertension (systolic blood pressure over 185 $\mathrm{mm} \mathrm{Hg}$ or diastolic blood pressure over $110 \mathrm{~mm} \mathrm{Hg}$ ); 4) baseline glucose concentration over $21.6 \mathrm{mmol} / \mathrm{L}$ or below $2.7 \mathrm{mmol} / \mathrm{L}$; 5) known coagulation factor deficiency, hemorrhagic diathesis, or oral anticoagulant treatment with an international normalized ratio over $1.7 ; 6$ ) treatment with heparin within 2 days with a partial thromboplastin time greater than double the laboratory normal and a baseline platelet count below $100 \times 10^{9} / \mathrm{L} ; 7$ ) serious allergy history (worse than rash) with regard to nitinol or other stent material; and 8) pregnancy.

\section{Randomization and Masking}

Eligible patients were enrolled at once after the first angiographic examination indicating adequacy of the occlusive lesion. They were randomly assigned at a 1:1 ratio to undergo MT using the RECO or the Solitaire FR device. The randomization sequence was generated by computer and stratified by both the site and the presenting NIHSS score ( $\leq 16$ vs $>16)$, with block sizes of four. Sequentially numbered, nontransparent, and hermetically sealed envelopes were used to ensure allocation concealment. The envelopes were prepared by an independent statistician, and the local sites did not know the randomization algorithm or the block size. Researchers could get randomization envelopes immediately, avoiding treatment delays. Enrollment in this research referred to the time when the randomization was finished and the designated device was displayed.

An independent central imaging core laboratory did not know the group allocation and made an assessment about the revascularization grade on the final angiogram as well as the hemorrhagic transformation outcome on MRI and CT. An independent clinical events committee did not know the group allocation and categorized every adverse event according to the severity and relevance to both the device and the operation. 


\section{Use of the RECO Clot Retriever}

Using angiography, the location and size of the occluded artery were assessed. With the aid of a 6-Fr guide catheter, the microcatheter was advanced distal to the thrombus by $2 \mathrm{~mm}$. The RECO clot retriever was transferred to the distal end of the microcatheter. The clot retriever was positioned so that its usable length portion would extend past each side of the thrombus in the vessel when fully deployed. After confirming its position, the stent was deployed and kept in place for at least 5 minutes. To extract the thrombus, the microcatheter and stent were slowly removed as a unit until the clot retriever and microcatheter were nearly withdrawn from the guide catheter, with continuous aspiration of the guide catheter using a syringe. If reperfusion was not achieved, the above process was repeated. The total number of attempts cannot exceed three times in the same artery.

\section{Procedures}

Endovascular treatment was conducted under conscious sedation and local anesthesia except in patients who could not cooperate because of severe aphasia. An angiogram was acquired before stent deployment to confirm the inclusion and exclusion criteria. Once a patient was allocated to the RECO or Solitaire FR group, the appropriately sized device was selected for use. At least one stent deployment was required within 8 hours after symptom onset. Up to three passes of the stent were permitted to obtain good recanalization (mTICI grade $\geq 2$ ). The operator could use rescue therapy after three passes. The definition of rescue therapy included the use of another MT device, intracranial angioplasty and/or stenting, etc. Before starting any rescue treatment, an angiogram was obtained to assess the primary efficacy endpoint after three passes of the device or at the end of the operation (if fewer than three passes). If rescue therapy was applied before three passes, the case was considered to have failed in terms of the primary efficacy endpoint. Follow-up evaluation was conducted at 1 day, 1 week, and 90 days after treatment. CT or MRI scans were acquired on day 1 postprocedure to check for hemorrhage. The NIHSS score as well as the modified Rankin Scale (mRS) score was obtained at the 1-day, 1-week, and 90-day follow-ups.

\section{Outcomes}

\section{Primary Efficacy Endpoint}

The mTICI scale was used to assess the degree of reperfusion. The primary efficacy endpoint was specified as good recanalization with an mTICI grade $\geq 2$ within three passes of the RECO or Solitaire stent without rescue therapy. Two professional neurointerventionalist readers from the independent core laboratory examined the angiograms and confirmed the mTICI grades. If these readers assigned divergent grades, another professional reader would adjudicate. A determination was made about the primary efficacy endpoint according to the grades.

\section{Secondary Efficacy Endpoints}

The prespecified secondary endpoints included the following: 1) functional independence with an $\mathrm{mRS}$ score $\leq$
2 at 90 days; 2) procedure duration, which was defined as the time from puncture to achieving an mTICI grade $\geq 2$ or to the final angiogram if an mTICI grade $\geq 2$ was not gained; and 3) 90-day all-cause mortality.

\section{Primary Safety Endpoints}

The primary safety endpoints consisted of the following: 1) any serious adverse device effect; 2) symptomatic intracerebral hemorrhage (sICH) within 24 hours $(-12 /+12$ hours) postprocedure, defined as ICH followed by clinical deterioration, i.e., an increase in the NIHSS score of $\geq 4$ points and considered to be the main cause of the neurological deterioration according to experts, or death; and 3) any serious adverse event (SAE; defined as cerebral palsy or death) within 24 hours after the procedure. All CT or MRI scans were evaluated by the independent core laboratory to discover and assess the hemorrhage's occurrence. Any hemorrhage, serious adverse device effect, or SAE reported in the trial was reviewed and judged by the clinical events committee.

\section{Statistical Analysis}

The initial hypothesis of the trial was that the proportion of the patients revascularized with the RECO stent would be noninferior to that revascularized with the Solitaire FR stent. This hypothesis was assessed with Blackwelder's method,${ }^{14}$ supposing unilateral $\alpha=0.025$ and the margin was $10 \%$. The null hypothesis $\left(\mathrm{H}_{0}\right)$ and alternative hypothesis $\left(\mathrm{H}_{\mathrm{A}}\right)$ in mathematical terms could be stated as follows: $\mathrm{H}_{0 \text { (noniferiority): }}: \mathrm{R}_{\mathrm{S}}-\mathrm{R}_{\mathrm{RECO}} \geq 0.10 ; \mathrm{H}_{\mathrm{A} \text { (noninferiority): }}$ : $\mathrm{R}_{\mathrm{S}}$ $-R_{R E C O}<0.10$, where $R_{S}$ and $R_{R E C O}$ are the revascularization rates noted after using the Solitaire FR stent and the RECO stent, respectively. The test included construction of the unilateral $97.5 \% \mathrm{CI}$ around the disparity in the revascularization rate $\left(R_{S}-R_{R E C O}\right)$. If the upper limit of the interval was below $0.10, \mathrm{H}_{0}$ was rejected and noninferiority was established. If noninferiority of the RECO stent was displayed, an analysis was made to use a two-tailed test at the significance level of 0.05 . A sensitivity analysis was performed to evaluate the impact of the differences between groups on baseline characteristics of the primary efficacy endpoint by means of univariate logistic regression of variables with a $p$ value less than or equal to 0.1 simplified to a final model by backward elimination, including variables that needed to be used as randomization stratification factors. For analyses of the secondary efficacy endpoints, the tests of noninferiority were unilateral, while all other tests were two-tailed. No adjustment of the resulting $\mathrm{p}$ values was made for multiplicity.

To establish the sample size, we took into account the Extending the Time for Thrombolysis in Emergency Neurological Deficits-Intra-Arterial (EXTEND-IA) trial ${ }^{4}$ results. The RECO and Solitaire FR devices were assumed to have a revascularization rate of $90 \%$ and $83 \%$, respectively. According to the assumptions, each group required 63 individuals in order to have $80 \%$ power of detecting noninferiority (unilateral $\alpha=0.025$ ) for a total of 126 patients. Because the success of revascularization was the only primary efficacy endpoint in the study, there was generally no problem regarding sample loss. It was decided to enroll 65 cases per group, for a total of 130 cases. 


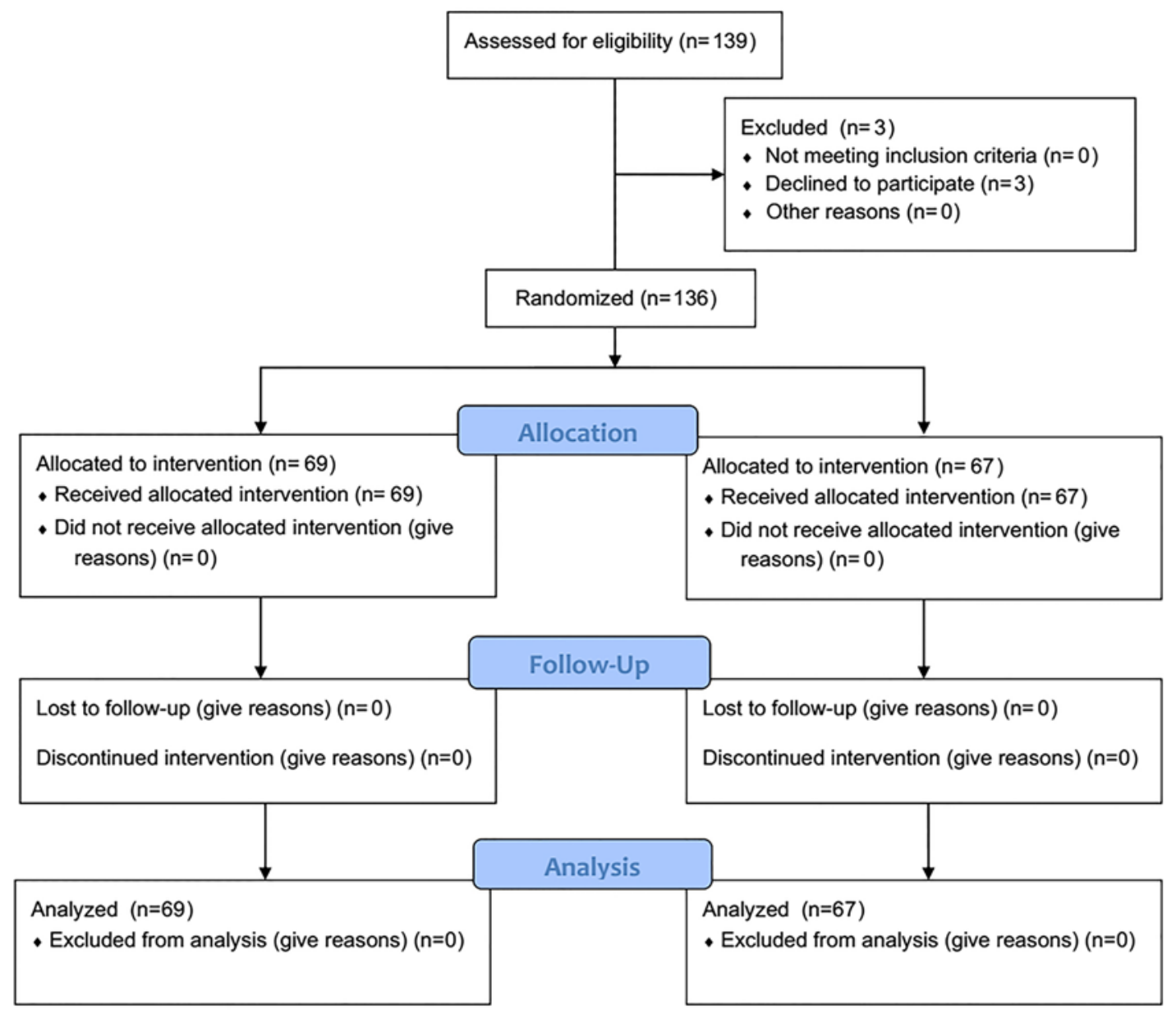

FIG. 2. Study flowchart. Figure is available in color online only.

We evaluated the primary safety outcomes and secondary endpoints with two-tailed tests. All enrolled patients assigned to either group were included in the intention-totreat population at random and were included in all analyses. Continuous variables were assessed by means of the Wilcoxon rank-sum test or two-sample t-test. Categorical variables were analyzed by means of Fisher's exact test. The standard error and log-rank tests were conducted by the Greenwood method to compare the two groups. The study data are reported as descriptive statistics. The results for all the patients in their assigned groups are summarized for all endpoints or measurements. A p $<0.05$ was considered statistically significant. Statistical analyses were conducted using SAS version 9.4 (SAS Institute Inc.).

\section{Role of the Funding Source}

The executive committee is composed of an academic steering committee, which was responsible for supervision of the design as well as the operation of the experiment, an academic principal investigator, and an academic lead interventional investigator. The sponsor of the trial was in charge of site and data management and security reporting. The sponsor conducted and monitored the trial in accordance with good clinical practice as well as China Food and Drug Administration (CFDA) regulations and concealed the results until the study was over and the database was locked. The sponsor was not involved in the analysis or interpretation of the data or the report writing. Statisticians as well as researchers were responsible for analyzing the data and adjusting the endpoints. The executive committee, which was responsible for report writing and data interpretation, had the right to access every statistic in this research and was ultimately responsible for the decision of the submission of the publication.

\section{Results}

The first site activation date of the trial was January 5 , 2014, and the enrollment closing date was May 7, 2016. The follow-up of the last enrolled patient was completed on August 7, 2016. During this period of time, 7 medical centers in China consented to participate in the trial and enrolled 136 patients, who were randomly assigned to either of the two treatment groups (69 to Solitaire and 67 to RECO; Fig. 2). Each enrolled patient underwent MT with a RECO or Solitaire stent. The primary endpoint was assessed for each enrolled patient, and no patients were lost to follow-up. 
TABLE 1. Baseline characteristics of 136 patients treated for AIS due to LVO

\begin{tabular}{|c|c|c|c|c|}
\hline Variable & Solitaire & RECO & Difference $(95 \% \mathrm{Cl})$ & p Value \\
\hline No. of patients & 69 & 67 & & \\
\hline Age in yrs & $67.19 \pm 10.23$ & $61.75 \pm 11.94$ & $5.45(1.71,9.18)$ & 0.0049 \\
\hline$\%$ males & $59 \%$ & $55 \%$ & $4.2 \%(-12.4 \%, 20.8 \%)$ & 0.7290 \\
\hline NIHSS score & $17.62 \pm 4.33$ & $17.43 \pm 4.58$ & $0.19(-1.31,1.69)$ & 0.8037 \\
\hline Systolic blood pressure in $\mathrm{mm} \mathrm{Hg}$ & $142.07 \pm 23.29$ & $141.36 \pm 20.36$ & $0.71(-6.65,8.08)$ & 0.8495 \\
\hline Diastolic blood pressure in $\mathrm{mm} \mathrm{Hg}$ & $86.01 \pm 14.50$ & $81.87 \pm 13.91$ & $4.15(-0.63,8.93)$ & 0.0911 \\
\hline Pre-stroke mRS score & & & & 0.2708 \\
\hline 0 & $96 \%$ & $93 \%$ & $3.1 \%(-4.8 \%, 11.0 \%)$ & \\
\hline 1 & $3 \%$ & $7 \%$ & $-4.6 \%(-12.0 \%, 2.9 \%)$ & \\
\hline 2 & $0 \%$ & $0 \%$ & $0.0 \%(0.0 \%, 0.0 \%)$ & \\
\hline 3 & $0 \%$ & $0 \%$ & $0.0 \%(0.0 \%, 0.0 \%)$ & \\
\hline 4 & $1 \%$ & $0 \%$ & $1.4 \%(-1.4 \%, 4.3 \%)$ & \\
\hline Intravenous rt-PA failure & $33 \%$ & $19 \%$ & $13.9 \%(-0.7 \%, 28.5 \%)$ & 0.0810 \\
\hline \multicolumn{5}{|l|}{ Medical history } \\
\hline Coronary artery disease & $22 \%$ & $22 \%$ & $-0.6 \%(-14.6 \%, 13.3 \%)$ & 0.9999 \\
\hline Atrial fibrillation & $46 \%$ & $43 \%$ & $3.1 \%(-13.6 \%, 19.8 \%)$ & 0.7334 \\
\hline Hypertension & $58 \%$ & $64 \%$ & $-6.2 \%(-22.6 \%, 10.1 \%)$ & 0.4861 \\
\hline Cardiac failure & $0 \%$ & $6 \%$ & $-6.0 \%(-11.6 \%,-0.3 \%)$ & 0.0562 \\
\hline Peripheral arterial disease & $1 \%$ & $0 \%$ & $1.4 \%(-1.4 \%, 4.3 \%)$ & 0.9999 \\
\hline Diabetes mellitus & $16 \%$ & $18 \%$ & $-2.0 \%(-14.6 \%, 10.6 \%)$ & 0.8214 \\
\hline Previous transient ischemic attack & $1 \%$ & $0 \%$ & $1.4 \%(-1.4 \%, 4.3 \%)$ & 0.9999 \\
\hline Previous ischemic stroke & $7 \%$ & $15 \%$ & $-7.7 \%(-18.2 \%, 2.8 \%)$ & 0.1792 \\
\hline Previous intracerebral hemorrhage & $0 \%$ & $0 \%$ & $0.0 \%(0.0 \%, 0.0 \%)$ & NA \\
\hline Smoking & $12 \%$ & $12 \%$ & $-0.3 \%(-11.2 \%, 10.5 \%)$ & 0.9999 \\
\hline \multicolumn{5}{|l|}{ Most proximal occlusion location } \\
\hline Internal carotid artery & $13 \%$ & $19 \%$ & $-6.4 \%(-18.7 \%, 6.0 \%)$ & 0.3576 \\
\hline$M_{1}$ segment & $67 \%$ & $58 \%$ & $8.5 \%(-7.8 \%, 24.7 \%)$ & 0.3763 \\
\hline$M_{2}$ segment & $9 \%$ & $13 \%$ & $-4.7 \%(-15.3 \%, 5.8 \%)$ & 0.4225 \\
\hline Basilar artery & $10 \%$ & $7 \%$ & $2.7 \%(-6.8 \%, 12.2 \%)$ & 0.7641 \\
\hline$V_{4}$ segment & $1 \%$ & $0 \%$ & $1.4 \%(-1.4 \%, 4.3 \%)$ & 0.9999 \\
\hline Posterior cerebral artery & $0 \%$ & $1 \%$ & $-1.5 \%(-4.4 \%, 1.4 \%)$ & 0.4926 \\
\hline Time from symptom onset to arterial puncture in mins & $272.32 \pm 103.54$ & $292.54 \pm 133.86$ & $-20.22(-60.37,19.93)$ & 0.3254 \\
\hline
\end{tabular}

$\mathrm{NA}=$ not applicable.

Values are expressed as the mean \pm standard deviation, unless indicated otherwise.

Table 1 shows the baseline demographic and clinical features of the two groups. There were no major divergences in the multiple variables between the two groups, including for presenting stroke severity (NIHSS score), blood pressure, medical history, and proximal occlusion location, except that the patients in the Solitaire group were older than those in the RECO group. The time from symptom onset to arterial puncture in the two groups was much the same. Thirty-three percent and $19 \%$ of the patients in the Solitaire and RECO groups, respectively, underwent intravenous rt-PA before the procedure $(\mathrm{p}=0.081)$.

Key time metrics and perioperative data are presented in Table 2. The mean times from symptom onset to first stent deployment and successful reperfusion were similar in the two groups. The NIHSS scores at 24 hours and 7 days after treatment were similar between the two groups as well. Eight patients in the Solitaire group acquired rescue treatment, while 9 patients in the RECO group did so $(p=0.7997)$. The difference in the mean number of passes between the two groups was not statistically significant. No significant difference was found in the rate of general anesthesia between the two groups.

\section{Primary Efficacy Endpoint}

The primary efficacy endpoint of good recanalization (mTICI grade $\geq 2 \mathrm{a}$ within three passes and no rescue therapy) was achieved in the RECO group at a similar rate as in the Solitaire group (91\% vs $87 \%$, respectively, $\mathrm{p}=$ 0.5861 ; Table 3). We also found no significant difference in the rate of reperfusion with an mTICI grade $\geq 2 \mathrm{~b}$ between the two groups ( $87 \%$ vs $75 \%, p=0.1272)$. 
TABLE 2. Key time metrics and perioperative data for 136 patients treated for AIS due to LVO

\begin{tabular}{|c|c|c|c|c|}
\hline Variable & Solitaire & RECO & Difference $(95 \% \mathrm{Cl})$ & $\mathrm{p}$ Value \\
\hline No. of patients & 69 & 67 & & \\
\hline Time from symptom onset to 1st stent deployment in mins & $302.54 \pm 118.79$ & $326.66 \pm 140.26$ & $-24.12(-67.76,19.52)$ & 0.2806 \\
\hline Time from symptom onset to $\mathrm{mTICl}$ grade $\geq 2$ reperfusion in mins & $362.26 \pm 133.76$ & $377.93 \pm 147.92$ & $-15.66(-63.04,31.71)$ & 0.5180 \\
\hline NIHSS score at $24 \mathrm{hrs}$ & $14.66 \pm 8.92$ & $12.88 \pm 9.07$ & $1.78(-1.28,4.84)$ & 0.2565 \\
\hline $\mathrm{mRS}$ score at $24 \mathrm{hrs}$ & & & & 0.5025 \\
\hline 0 & $2.9 \%$ & $3.0 \%$ & & \\
\hline 1 & $4.3 \%$ & $4.5 \%$ & & \\
\hline 2 & $7.2 \%$ & $6.0 \%$ & & \\
\hline 3 & $1.4 \%$ & $9.0 \%$ & & \\
\hline 4 & $27.5 \%$ & $32.8 \%$ & & \\
\hline 5 & $55.1 \%$ & $43.3 \%$ & & \\
\hline 6 & $1.4 \%$ & $1.5 \%$ & & \\
\hline NIHSS score at 7 days & $11.47 \pm 10.00$ & $9.11 \pm 9.93$ & $2.36(-1.04,5.76)$ & 0.1755 \\
\hline $\mathrm{mRS}$ score at 7 days & & & & 0.3573 \\
\hline 0 & $4.3 \%$ & $10.4 \%$ & & \\
\hline 1 & $11.6 \%$ & $11.9 \%$ & & \\
\hline 2 & $7.2 \%$ & $9.0 \%$ & & \\
\hline 3 & $10.1 \%$ & $14.9 \%$ & & \\
\hline 4 & $18.8 \%$ & $25.4 \%$ & & \\
\hline 5 & $43.5 \%$ & $26.9 \%$ & & \\
\hline 6 & $4.3 \%$ & $1.5 \%$ & & \\
\hline Thrombectomy passes & $2.09 \pm 0.78$ & $1.90 \pm 0.76$ & $0.19(-0.07,0.45)$ & 0.1503 \\
\hline Use of rescue treatment & $12 \%(8 / 69)$ & $13 \%(9 / 67)$ & $-1.8 \%(-13.0 \%, 9.3 \%)$ & 0.7997 \\
\hline General anesthesia & $18.8 \%(13 / 69)$ & $16.4 \%(11 / 67)$ & $2.4 \%(-10.4 \%, 15.2 \%)$ & 0.7113 \\
\hline
\end{tabular}

\section{Primary Safety Endpoints}

No serious adverse device effects occurred in either group. One patient $(1.5 \%)$ in the RECO group and 5 patients $(7.2 \%)$ in the Solitaire group had sICH $(\mathrm{p}=0.1027$; Table 3). The rates of death and cerebral palsy within 24 hours did not differ between the groups.

\section{Secondary Efficacy Endpoints}

The distribution of mRS scores at 90 days in each group is shown in Fig. 3. Compared with the Solitaire group, the RECO group showed a notable trend toward better long-term functional outcomes $(46 \%$ vs $63 \%, p=0.0609$; Table 3), though the difference between the two groups was not statistically significant. No significant difference was found in the rate of 90-day mortality between the two groups $(23 \%$ vs $13 \%$, respectively, $\mathrm{p}=0.1848)$. Procedure duration was much the same in the two groups.

\section{Discussion}

This multicenter, prospective RCT suggests that the RECO FR device has the same advantage in successful recanalization in AIS due to LVO as the Solitaire FR stent retriever, with similar rates of sICH, SAEs, functional independence, and 90-day mortality.

Functional independence (mRS score $\leq 2$ ) at 90 days is considered to be the gold standard for assessing the clinical outcome of intraarterial treatment for AIS. More complete reperfusion of ischemia has been related to better clinical outcomes. ${ }^{15}$ Among the various methods of recanalization, MT with a stent retriever is widely used, and this technique improves recanalization rates and reduces the incidence of periprocedural complications. ${ }^{12}$ The Solitaire FR device was the first stent retriever confirmed to be safe and efficient for LVO in the SWIFT trial,,$^{10}$ which was the first AIS study to directly and randomly compare one endovascular recanalization technique (Solitaire device) with another (Merci device). In the SWIFT trial, the successful recanalization rate (TICI grade $2 \mathrm{a}$ or better) in the Solitaire stent group was higher than that in the Merci group (61\% vs 24\%). Solitaire devices have been assessed in many subsequent trials and prospective registries, some of which have reported higher rates of reperfusion than were achieved in the SWIFT study. The SWIFT PRIME trial (Solitaire With the Intention For Thrombectomy as Primary Endovascular Treatment trial) and EXTEND-IA trial with Solitaire stents achieved high successful recanalization rates of $88 \%$ and $86 \%$, respectively, using the definition of successful recanalization TICI grade $\geq 2 b{ }^{4,5}$ In addition, in the above three trials, Solitaire devices, but not the Merci devices or medical therapies, were related to higher rates of 90-day functional independence.

Note that the RECO stent is a closed-loop design like the Solitaire stent. As the first clot retriever in China, the 
TABLE 3. Efficacy and safety endpoints among 136 patients treated for AIS due to LVO

\begin{tabular}{|c|c|c|c|c|}
\hline Endpoint & Solitaire & RECO & Difference $(95 \% \mathrm{Cl})$ & $\mathrm{p}$ Value \\
\hline \multicolumn{5}{|l|}{ Primary efficacy endpoint } \\
\hline Successful recanalization: $\mathrm{mTICI}$ grade $\geq 2 a$ & $87 \%(60 / 69)$ & $91 \%(61 / 67)$ & $-4.1 \%(-14.6 \%, 6.4 \%)$ & 0.5861 \\
\hline Successful recanalization: $\mathrm{mTICI}$ grade $\geq 2 b$ & $75 \%(52 / 69)$ & $87 \%(58 / 67)$ & $-11.2 \%(-24.2 \%, 1.8 \%)$ & 0.1272 \\
\hline $\mathrm{mTICl}$ grade 0 & $7 \%(5 / 69)$ & $2 \%(1 / 66)$ & $5.7 \%(-1.1 \%, 12.5 \%)$ & 0.2089 \\
\hline $\mathrm{mTICl}$ grade 1 & $6 \%(4 / 69)$ & $6 \%(4 / 66)$ & $-0.3 \%(-8.2 \%, 7.7 \%)$ & 1.0000 \\
\hline $\mathrm{mTICl}$ grade $2 \mathrm{a}$ & $12 \%(8 / 69)$ & $5 \%(3 / 66)$ & $7.0 \%(-2.0 \%, 16.1 \%)$ & 0.2082 \\
\hline $\mathrm{mTICl}$ grade $2 \mathrm{~b}$ & $22 \%(15 / 69)$ & $39 \%(26 / 66)$ & $-17.7 \%(-32.9 \%,-2.4 \%)$ & 0.0388 \\
\hline $\mathrm{mTICl}$ grade 3 & $54 \%(37 / 69)$ & $48 \%(32 / 66)$ & $5.1 \%(-11.7 \%, 22.0 \%)$ & 0.6071 \\
\hline \multicolumn{5}{|l|}{ Primary safety endpoints } \\
\hline Serious adverse device effects & 0 & 0 & 0 & NA \\
\hline slCH w/in 24 hrs & $7.2 \%(5 / 69)$ & $1.5 \%(1 / 67)$ & $5.8 \%(-1.1 \%, 12.7 \%)$ & 0.1027 \\
\hline Death w/in 24 hrs & $1.4 \%(1 / 69)$ & $1.5 \%(1 / 67)$ & $-0.0 \%(-4.1 \%, 4.0 \%)$ & 0.9999 \\
\hline Cerebral palsy w/in 24 hrs & $0 \%(0 / 69)$ & $4.5 \%(3 / 67)$ & $-4.5 \%(-9.4 \%, 0.5 \%)$ & 0.1168 \\
\hline \multicolumn{5}{|l|}{ Secondary efficacy endpoints } \\
\hline 90-day good outcome (mRS score $0-2$ ) & $46 \%(32 / 69)$ & $63 \%(42 / 67)$ & $-16.3 \%(-32.8 \%, 0.2 \%)$ & 0.0609 \\
\hline Procedure time from puncture to $\mathrm{mTICI}$ reperfusion grade $\geq 2$ in mins & $89.94 \pm 53.34$ & $85.39 \pm 47.01$ & $4.55(-12.36,21.47)$ & 0.5986 \\
\hline All-cause mortality during 90 days & $23 \%(16 / 69)$ & $13 \%(9 / 67)$ & $9.8 \%(-3.1 \%, 22.6 \%)$ & 0.1848 \\
\hline
\end{tabular}

RECO stent focuses on the cerebrovascular diseases of East Asian people; for example, a 3-mm device is part of the product profile to handle smaller blood vessels. The design of the RECO device has the following advantages over Solitaire. First, RECO uses mechanical fastening to strengthen the connection between the stent and the push wire, which improves push ability and the safety of withdrawing the device. Second, the struts of the RECO stent have been optimized in their design. The radial force is slightly greater than that with the Solitaire stent, and the success rate of recanalization seems to be higher. Moreover, the RECO stent has more options in diameter and length, making it suitable for intracranial vessels of different sizes. In our trial, we defined the reperfusion mTICI grade $\geq 2 \mathrm{a}$ as successful recanalization, which was the sole primary efficacy endpoint. We compared the efficacy of the RECO and Solitaire devices. The rate of reperfusion with an mTICI grade $\geq 2 \mathrm{a}$ with the RECO device was $91 \%$, whereas the rate of reperfusion with an mTICI grade $\geq$ 2 b was $87 \%$, rates in accordance with values in the lit- erature, which vary from $66.7 \%$ to $94.8 \%{ }^{16-20}$ Functional independence at 90 days was attained in $63 \%$ of patients in the RECO group, again within the range described in the most optimistic studies $(53 \%-71 \%)$. No significant difference was found in the rate of recanalization or functional independence between the RECO and Solitaire groups, confirming the efficacy of the device.

Saber et al. $^{13}$ performed a network meta-analysis of randomized data to compare different MT devices and strategies. They found that the Solitaire FR device showed the highest probability of being the most secure stroke intervention associated with significantly lower rates of sICH and catastrophic outcomes, as compared to medical treatment and the Merci and Trevo devices. In our trial, sICH occurred in only 1 patient in the RECO group, fewer than the 5 patients in the Solitaire group, with no significant difference between the two groups. Meanwhile, a large meta-analysis of observational studies revealed a significantly lower rate of mortality with respect to the use of the Solitaire device compared to the

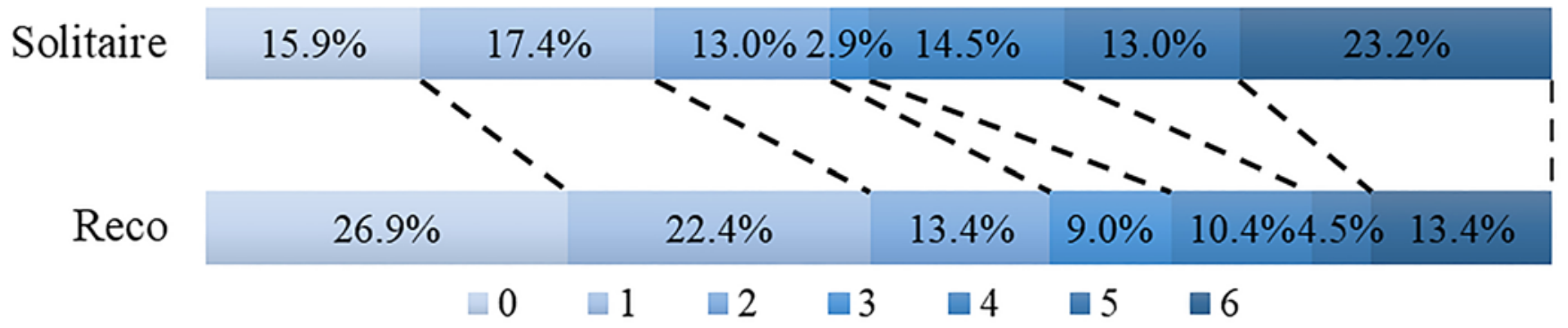

FIG. 3. Distribution of $\mathrm{mRS}$ scores at 90 days after treatment in the two treatment groups. Data were available for 69 patients in the Solitaire group and 67 patients in the RECO group. Figure is available in color online only. 
Trevo device. ${ }^{12}$ However, our trial indicates that the rate of 90-day mortality was lower in the RECO group than in the Solitaire group, although with no statistically significant difference between the two. In addition, there were no serious adverse device effects in either group. These findings are beyond our expectations, and we suspect that they are mostly related to the experience of the operators. Three patients in the RECO group had cerebral palsy within 24 hours due to the large infarction in the cerebral hemisphere, while no cases of cerebral palsy occurred in the Solitaire group.

Note that time is an independent factor for prognosis at 90 days in AIS, surpassing the benefit of good recanalization..$^{21}$ Numerous passes and lengthy procedures predict the likelihood of a good clinical outcome. ${ }^{22-24}$ In our trial, the times from symptom onset to arterial puncture, first stent deployment, and successful reperfusion were similar between the RECO and Solitaire groups. There was no significant difference in the mean number of passes or procedure duration between the groups.

There were some limitations to our study. First, because it is the first randomized AIS trial in China to compare one endovascular recanalization technique with another since 2014, we defined successful recanalization as an mTICI grade of 2a or better, with reference to the SWIFT ${ }^{10}$ and TREVO $2^{11}$ trials. However, in most of the recent RCTs, an mTICI grade $\geq 2 b$ was defined as successful revascularization at the end of the procedure. Fortunately, we analyzed the rate of an mTICI grade $\geq 2 b$ being achieved within three passes of the two devices and found no significant difference between the two groups, further confirming the efficacy of the RECO device. Second, the study compared the RECO stent retriever with the Solitaire device only. Comparisons were not made with aspiration systems or other emerging stent retrievers. Further studies will be needed to describe the clinical efficacy and safety of the RECO device relative to these alternatives.

\section{Conclusions}

The RECO stent retriever is effective and safe as an MT device for AIS due to LVO.

\section{References}

1. Berkhemer OA, Fransen PS, Beumer D, et al. A randomized trial of intraarterial treatment for acute ischemic stroke. $N$ Engl J Med. 2015;372(1):11-20.

2. Jovin TG, Chamorro A, Cobo E, et al. Thrombectomy within 8 hours after symptom onset in ischemic stroke. $N$ Engl J Med. 2015;372(24):2296-2306.

3. Puñal-Riobóo J, Atienza G, Blanco M. Safety and efficacy of mechanical thrombectomy using stent retrievers in the endovascular treatment of acute ischaemic stroke: a systematic review. Intervent Neurol. 2015;3(3-4):149-164.

4. Campbell BC, Mitchell PJ, Kleinig TJ, et al. Endovascular therapy for ischemic stroke with perfusion-imaging selection. N Engl J Med. 2015;372(11):1009-1018.

5. Saver JL, Goyal M, Bonafe A, et al. Stent-retriever thrombectomy after intravenous t-PA vs. t-PA alone in stroke. N Engl J Med. 2015;372(24):2285-2295.

6. Khatri P, Yeatts SD, Mazighi M, et al. Time to angiographic reperfusion and clinical outcome after acute ischaemic stroke: an analysis of data from the Interventional
Management of Stroke (IMS III) phase 3 trial. Lancet Neurol. 2014;13(6):567-574.

7. Yi HJ, Lee DH, Kim SU. Effectiveness of Trevo stent retriever in acute ischemic stroke: comparison with Solitaire stent. Medicine (Baltimore). 2018;97(20):e10747.

8. Bourcier R, Abed D, Piotin M, et al. Multicenter initial experience with the EmboTrap device in acute anterior ischemic stroke. J Neuroradiol. 2018;45(4):230-235.

9. Zaidat OO, Bozorgchami H, Ribó M, et al. Primary results of the multicenter ARISE II study (Analysis of Revascularization in Ischemic Stroke With EmboTrap). Stroke. 2018;49(5):1107-1115.

10. Saver JL, Jahan R, Levy EI, et al. Solitaire flow restoration device versus the Merci Retriever in patients with acute ischaemic stroke (SWIFT): a randomised, parallel-group, non-inferiority trial. Lancet. 2012;380(9849):1241-1249.

11. Nogueira RG, Lutsep HL, Gupta R, et al. Trevo versus Merci retrievers for thrombectomy revascularisation of large vessel occlusions in acute ischaemic stroke (TREVO 2): a randomised trial. Lancet. 2012;380(9849):1231-1240.

12. Walcott BP, Boehm KM, Stapleton CJ, et al. Retrievable stent thrombectomy in the treatment of acute ischemic stroke: analysis of a revolutionizing treatment technique. J Clin Neurosci. 2013;20(10):1346-1349.

13. Saber H, Rajah GB, Kherallah RY, et al. Comparison of the efficacy and safety of thrombectomy devices in acute stroke: a network meta-analysis of randomized trials. J Neurointerv Surg. 2018;10(8):729-734.

14. Blackwelder WC. "Proving the null hypothesis" in clinical trials. Control Clin Trials. 1982;3(4):345-353.

15. Saver JL, Goyal M, van der Lugt A, et al. Time to treatment with endovascular thrombectomy and outcomes from ischemic stroke: a meta-analysis. JAMA. 2016;316(12):12791288.

16. Prothmann S, Schwaiger BJ, Gersing AS, et al. Acute Recanalization of Thrombo-Embolic Ischemic Stroke with pREset (ARTESp): the impact of occlusion time on clinical outcome of directly admitted and transferred patients. $J$ Neurointerv Surg. 2017;9(9):817-822.

17. Kabra R, Phillips TJ, Saw JL, et al. Mechanical thrombectomy for anterior circulation stroke: 5-year experience in a statewide service with differences in pretreatment time metrics across two hospitals sites. $J$ Neurointerv Surg. 2017;9(6):535-540.

18. Minnerup J, Wersching H, Teuber A, et al. Outcome after thrombectomy and intravenous thrombolysis in patients with acute ischemic stroke: a prospective observational study. Stroke. 2016;47(6):1584-1592.

19. Raoult H, Redjem H, Bourcier R, et al. Mechanical thrombectomy with the ERIC retrieval device: initial experience. J Neurointerv Surg. 2017;9(6):574-577.

20. Hopf-Jensen S, Preiß M, Marques L, et al. Impact and effectiveness of dual aspiration technique in stent-assisted mechanical thrombectomy: recent improvements in acute stroke management. Cardiovasc Intervent Radiol. 2016;39(11):1620-1628.

21. Goyal M, Menon BK, van Zwam WH, et al. Endovascular thrombectomy after large-vessel ischaemic stroke: a metaanalysis of individual patient data from five randomised trials. Lancet. 2016;387(10029):1723-1731.

22. Marder VJ, Chute DJ, Starkman S, et al. Analysis of thrombi retrieved from cerebral arteries of patients with acute ischemic stroke. Stroke. 2006;37(8):2086-2093.

23. Costalat V, Lobotesis K, Machi P, et al. Prognostic factors related to clinical outcome following thrombectomy in ischemic stroke (RECOST study). 50 patients prospective study. Eur J Radiol. 2012;81(12):4075-4082.

24. Bourcier R, Brecheteau N, Costalat V, et al. MRI quantitative $\mathrm{T} 2 *$ mapping on thrombus to predict recanalization after 
endovascular treatment for acute anterior ischemic stroke. $J$ Neuroradiol. 2017;44(4):241-246.

\section{Disclosures}

The study was funded by Minitech Medical.

\section{Author Contributions}

Conception and design: Peng, Ke, Yong Zhang, Wang, M Zhang. Acquisition of data: Peng, Cao, H Lin, M Lin, Ke, Yunfeng Zhang, Yong Zhang, Zheng, Wang, M Zhang, Xuan. Analysis and interpretation of data: Cao, Chen. Drafting the article: Cao. Criti- cally revising the article: all authors. Reviewed submitted version of manuscript: Peng, Cao, Yunfeng Zhang. Approved the final version of the manuscript on behalf of all authors: Peng. Statistical analysis: Cao. Administrative/technical/material support: all authors. Study supervision: Peng, H Lin, Ke, Yong Zhang, Chen, Wang, M Zhang, Xuan.

\section{Correspondence}

Ya Peng: The First People's Hospital of Changzhou/The Third Affiliated Hospital of Soochow University, Changzhou, Jiangsu Province, China. neuropengya@sina.com. 